\title{
Transfusion of stored platelets: balancing risks and product availability
}

This article was published in the following Dove Press journal:

International Journal of Clinical Transfusion Medicine

II November 2016

Number of times this article has been viewed

\section{Cécile Aubron ${ }^{1,2}$ \\ Andrew W Flint 2,3 \\ Yves Ozier ${ }^{4}$ \\ Zoe McQuilten ${ }^{2,5}$}

'Medical Intensive Care Unit, Centre Hospitalier et Universitaire de Brest site La Cavale Blanche-Université de Bretagne Occidentale, Brest, France; ${ }^{2}$ Department of Epidemiology and Preventive Medicine, The Australian and New Zealand Intensive Care Research Centre, Monash University, Melbourne, VIC, Australia; ${ }^{3}$ Royal Australian Navy, Australian Defence Force, Canberra, ACT, Australia; ${ }^{4}$ Department of Anesthesiology, Centre Hospitalier et Universitaire de Brest site La Cavale Blanche-Université de Bretagne Occidentale, Brest, France; ${ }^{5}$ Department of Epidemiology and Preventive Medicine, Transfusion Research Unit, Monash University, Melbourne, VIC, Australia
Correspondence: Cécile Aubron Réanimation Médicale, CHRU de Brest site La Cavale Blanche, Bvd Tanguy Prigent 29609 Brest Cedex, France Tel +3329834 7l8I

Fax +33 298347965

Email cecile.aubron@chu-brest.fr
Abstract: Platelet (PLT) transfusions are used for both treatment of bleeding and preventing bleeding in patients with thrombocytopenia and/or functionally abnormal PLTs. To ensure the efficacy of transfused PLTs, they must be stored at room temperature. Due to this storage requirement, PLTs have a short shelf life compared with other blood components of up to 5-7 days to minimize the risk of bacterial contamination. This short shelf life can lead to PLT shortages at times of increased demand or reduced supply and also higher wastage due to expiry. Increasing the PLT storage duration has the potential to improve PLT availability at times of altered demand or supply. However, in addition to the risk of bacterial contamination, functional and biochemical changes occur in PLTs and their storage medium during storage that may lead to PLT activation, changes in efficacy, and adverse events. This narrative review analyzes the issues around PLT inventory management and potential risks of stored PLTs. Although in vitro studies show accumulation of reactive biomarkers in PLT units, the clinical significance of these changes remains uncertain and there is currently no evidence for an association between PLT storage duration and adverse events. Furthermore, PLTs processed and stored for up to 7 days in defined conditions could be as safe as fresh PLTs. Large prospective studies are warranted to better evaluate the safety of PLTs with extended storage and to define the best practice of optimizing PLT inventory management while maintaining PLT transfusion safety.

Keywords: platelet storage, storage lesion, PLT inventory management, transfusion adverse events

\section{Introduction}

Platelet (PLT) transfusions are routinely used for the treatment of bleeding in patients with thrombocytopenia or functionally abnormal PLTs (therapeutic transfusion) and to minimize the risk of bleeding in patients with thrombocytopenia (prophylactic transfusion). ${ }^{1,2}$ PLT transfusion has been associated with adverse events that can be categorized as follows: 1) adverse events immediately related and/or clearly attributable to PLT transfusion (ie, febrile nonhemolytic transfusion reaction, allergic reactions, transfusion-associated sepsis, transfusion-related acute lung injury (TRALI), alloimmunization, and posttransfusion purpura) and 2) adverse events reported to be associated with PLT transfusion, although causation and mechanisms have not been established (ie, sepsis and organ failure). ${ }^{3-6}$ For the second group of adverse events, proposed mechanisms include accumulation of pro-inflammatory substances ${ }^{7,8}$ and transfusion-related immune modulation. ${ }^{9}$

Both recipient and PLT component characteristics may play a key role in adverse events following PLT transfusion. Storage of blood components is associated with 
biochemical and structural changes which may be deleterious for recipients, ${ }^{10}$ as well as an increasing risk of bacterial contamination. It is important to understand the changes that occur in PLTs during storage and the implications of these changes for PLT efficacy and safety because 1) the short shelf life of PLTs poses significant challenges to PLT inventory management, especially in smaller hospitals or remote regions where it is difficult to maintain PLT inventories to support clinical services and at times of increased demand; 2) there is an evidence that the demand for PLTs is increasing worldwide; ${ }^{11}$ 3) changes in PLT screening and introduction of pathogen reduction technologies (PRTs) over recent decades may have altered the risk of bacterial contamination over storage duration; and 4) potential associations between duration of PLT storage and adverse events secondary to the accumulation of bioreactive substances have been reported. ${ }^{8,12,13}$

This narrative review first explores issues around PLT inventory management; second, it describes PLT production process and PLT storage lesion; and finally summarizes the potential risks associated with transfusion of PLTs stored for different durations.

\section{Impact of PLT storage duration on inventory management}

The short shelf life of liquid PLTs poses challenges to blood and health services with regard to inventory management, with a balance required to ensure that PLTs are available when and where needed while, at the same time, minimizing PLT wastage. There is therefore a necessary trade-off between shortage and wastage. Over $20 \%$ of PLTs may be wasted due to outdating. This varies both within and between countries, and also varies over the course of a year. ${ }^{14}$ In Europe, the reported proportion of PLTs that are discarded due to expiry varies from $1 \%$ to $20 \%,{ }^{15}$ and outdating has been reported up to $21 \%$ in Australia. ${ }^{16}$ Due to the short shelf life, PLT availability may be limited in small or remote hospitals, where low rates of demand do not justify the associated wastage with maintaining an inventory of PLTs. In addition, ensuring PLT availability in the event of infectious outbreaks or other threats to the blood supply is particularly challenging due to the short shelf life. ${ }^{17}$

Adding to the challenges for blood services in managing PLT inventory is the increasing demand for PLTs that has been observed internationally. There has been an increase in the demand for PLTs, with a reported increase of 15\%-16\% in the United Kingdom over the past decade after taking into account changes in population. ${ }^{11}$ This equates to a raise from 4.2 to 4.9 components per 1,000 inhabitants being issued in
2002 compared with 2012. Similar changes have also been shown for other European countries. ${ }^{11}$ Possible causes for this rising demand include an increasing and aging population, increased prevalence of hematological malignancies, and changes in management of hematological malignancies, resulting in requirement for greater PLT support. ${ }^{11}$

Increasing PLT production may not address all issues regarding PLT availability, particularly access in remote and regional areas, and may lead to more wastage and higher cost. Furthermore, PLT wastage raises ethical issues, for both donors and recipients. Other strategies that are being considered to improve PLT availability include increasing PLT storage duration from 5 to 6 or 7 days, which has already been adopted during periods of shortage by some countries ${ }^{18,19}$ and alternatives to liquid-stored PLTs. ${ }^{20}$

Extending PLT shelf life may have the benefit of improving supply while also reducing wastage. To illustrate this, the level of wastage was reported to increase in the US following the U.S. Food and Drug Administration's decision to shorten the shelf life from 7 to 5 days in $1986 .{ }^{21} \mathrm{~A}$ study of a single hospital's experience reported a significant decrease in wastage during a 6-month period when the shelf life of PLTs was increased again from 5 to 7 days. ${ }^{22}$ Several papers in the field of Operations Research have attempted to predict the impact on wastage by extending the shelf life. ${ }^{23}$ Haijema et al set a combined stochastic dynamic programming and simulation model to develop an ordering strategy to decrease wastage and shortages. ${ }^{23}$ The authors found that if the shelf life was extended, there would be a further decrease in wastage. ${ }^{23}$ When applying this model to a Dutch blood bank, de Kort et al found a substantial decrease in wastage and shortage rates. ${ }^{14}$

Alternatives to liquid-stored PLTs being explored may address the problem of supplying PLTs in smaller and remote health services that cannot justify maintaining PLT inventories due to low demand and high wastage. Cryopreserved PLTs have been used in the military setting, where provision of PLTs is challenging and maintaining an inventory of liquid-stored PLTs is not feasible. ${ }^{24}$ Initially developed by the US Navy in the 1970s, the cryopreservation method has been modified over time, and more recently used by the Dutch military in Bosnia and Afghanistan..$^{20,25}$ While cryopreserved PLTs may be advantageous in remote civilian health services, there are few studies evaluating efficacy and safety of cryopreserved PLTs outside the military setting. One controlled clinical trial of 73 cardiac surgery patients randomized to cryopreserved or liquid-stored PLTs has been undertaken. This trial showed no difference in adverse effects with less 
blood loss in the group that received cryopreserved PLTs. ${ }^{26}$ Although encouraging, further studies powered for safety outcomes are required, such as the clinical trial in cardiac surgery underway in Australia (Australian New Zealand Clinical Trials Registry ACTRN12612001261808). The use of lyophilized PLTs may also be an alternative to liquidstored PLTs in bleeding patients; however, issues related to efficacy, safety, and antigenicity persist, and further clinical studies are required before this can be introduced into practice. $^{20,27}$

While there are alternatives to liquid-stored PLTs being investigated and used in some settings, the remainder of this review will focus on the processing and storage lesion of liquid PLTs.

\section{PLT processing and storage}

PLT can be prepared from a single donor by apheresis or from whole blood using the buffy-coat or PLT-rich plasma methods. Four to six whole blood PLT concentrates are pooled to create an adult therapeutic PLT dose, while a therapeutic dose of PLT apheresis comes from a single donor. PLTs require specific and careful methods in their processing to maintain efficacy and to ensure safety. PLTs must be stored at room temperature with constant agitation, as cool temperature affects their efficacy. However, a storage temperature at $22^{\circ} \mathrm{C}$ promotes bacterial growth and increases the risk of bacterial contamination.

Besides these specific requirements for PLT storage, there is a high variability in PLT processing internationally, including production methods, type of additive solution, use and type of pathogen inactivation method, and storage duration. ${ }^{28}$

PLT storage media have changed over time, improving PLT transfusion safety. Initially, PLTs were only stored in plasma. However, the introduction of PLT additive solution has reduced the volume of plasma transfused and incidence of adverse reactions related to plasma transfusion, as well as enabled longer storage duration. ${ }^{29,30}$

Bacterial screening, usually performed in the first 30 hours after PLT preparation, has been largely implemented to prevent transfusion-associated sepsis due to administration of a bacterially contaminated PLT unit. ${ }^{31}$ Bacterial screening does not alter PLT characteristics; however, it has a low sensitivity, especially when the bacterial load is low. To offset this limitation, a second testing for bacterial contamination just prior to PLT issue has been proposed but remains rarely used. PRTs were introduced two decades ago and are increasingly being used by transfusion services. ${ }^{31}$ PRTs include inactivation by amotosalen or riboflavin (vitamin $\mathrm{B}_{2}$ ) that is added into the PLT unit, in association with ultraviolet irradiation. PRTs inactivate not only pathogens, including bacteria, some viruses, and protozoa, but also residual leukocytes.

Altogether, improvements in PLT storage methods and strategies to reduce risk of bacterial contamination have offered the possibility of extending PLT storage duration to 7 days. ${ }^{7,28,32}$ More prolonged storage durations have also been investigated. ${ }^{33}$ Nonetheless, questions around stored PLT safety and quality persist.

Several factors that influence PLT quality have been identified, including PLT storage medium characteristics, PLT bag (size and plastic material), PLT concentration, storage temperature, donor characteristics, and storage duration. ${ }^{29,34,35}$ During storage, PLTs undergo biochemical, morphological, and functional changes, which are referred to as the storage lesion. ${ }^{36,37}$ These changes occur despite optimization of storage conditions and the addition of key components, including glucose, calcium, bicarbonate, and magnesium $^{29}$ and they result in decreased PLT viability and increased PLT activation.

At room temperature, PLT metabolism remains active, requiring optimal oxygen and glucose concentrations. A shortage in either of these substrates leads to anaerobic metabolism, with lactate production and decrease in $\mathrm{pH}$, which contributes to the PLT storage lesion and impacts PLT viability. ${ }^{37}$ Therefore, any factors that may decrease oxygen will lead to anaerobic metabolism and acidification of the storage medium. The absence of agitation, high PLT concentration in the bag, a container bag too thick to allow gas exchange with the atmosphere, or too small or not enough gas-permeable surface area will result in a decrease in oxygen and PLT storage lesion. Additional parameters have been identified that may lead to PLT structural and functional changes, including ultraviolet light used for PRTs, temperature $<20^{\circ} \mathrm{C}$, and hyperbaric state. ${ }^{36-38}$ For instance, PRTs impact on PLT metabolism and viability ${ }^{39,40}$ and could therefore challenge the safety of stored PRT-treated PLTs.

In addition to PLT viability and efficacy issues, PLT activation occurs during the storage period. From the second day of storage, PLTs express activation biomarkers that could interact with mononuclear cells or endothelial cells, enhancing inflammatory responses in PLT recipients. ${ }^{12}$ Again, characteristics of the storage medium do impact on PLT activation. ${ }^{30}$

However, correlation between storage lesion and in vivo recovery is inconsistent. ${ }^{41}$ Similarly, the clinical impact of the PLT storage lesion in terms of safety has not been well assessed and remains unknown. 


\section{Exploring safety of stored PLTs}

Prior to extending PLT storage duration in order to improve PLT availability, it is crucial to anticipate and evaluate the risks, safety, and benefits of such a change, because unjustified changes may have deleterious consequences in recipients. On the other hand, limiting PLT storage duration may lead to unnecessary PLT wastage and an increase in PLT shortages.

Studies published two and three decades ago have suggested that prolonged PLT storage duration could have adverse clinical effects. ${ }^{42-45}$ However, methods in PLT manufacturing, preparation, and storage have changed over the last 20 years, challenging the generalizability of these study findings.

\section{Transfusion-associated sepsis due to administration of bacterially contaminated PLTs}

Despite systematic screening for bacterial contamination and implementation of PRTs in some countries, bacterial infection remains the leading infectious risk of PLT transfusion. In France, $\sim 2.2$ of every 100,000 PLT units lead to clinical bacterial infections with a fatality rate of $1: 200,000 .^{7,46,31}$ Between 127 and 1,885 per million units are contaminated based on culture results in the US. ${ }^{34}$ The incidence of septic transfusion reactions secondary to PLT administration is likely to be underestimated due to lack of sensitivity and specificity of the diagnostic criteria used and reliance mainly on passive surveillance. ${ }^{47}$ Improving prevention, diagnosis, and reporting of transfusion-associated sepsis should be a priority to ensure PLT safety, especially if considering changes to PLT storage duration.

\section{Transfusion-related adverse events}

The available data suggest that PLT storage duration does not impact on the occurrence of transfusion-related adverse events (TRAE) ${ }^{5,32}$ In a large post hoc analysis of the platelet dose randomized controlled trial that analyzed 5,034 prophylactic transfusions to 1,102 hematologically stable patients, ${ }^{5,48}$ PLT storage duration ( $0-2,3$, and 4 days compared to 5 days) was not associated with TRAE based on the Common Toxicity Criteria for Adverse Events (version 3) definition, whether considered all together or individually (fever, allergic/hypersensitivity, and any grade 2 or higher TRAE) ${ }^{5,48}$ This study had a large sample size and robust statistical analysis; however, exclusion of patients receiving consecutive PLT units may have resulted in selection bias. A recent study found that PLT storage duration of riboflavin-based PRT-treated PLT did not have an impact on TRAE occurrence when comparing PLTs stored for 6-7 days with PLT stored for $\leq 5$ days. ${ }^{32}$

\section{Transfusion-related acute lung injury}

PLT transfusion, as transfusion of any blood component, can cause TRALI. However, the potential role of PLT storage duration in TRALI pathogenesis after PLT administration remains unknown. In an in vivo transfusion model, Vlaar et al showed that transfusion of aged PLTs induces lung inflammation in healthy rats and is responsible for pulmonary and systemic coagulopathy in pretreated rats with lipopolysaccharide. ${ }^{8}$ The soluble cluster of differentiation 40 ligands that get accumulated over the storage period can activate leukocytes and could be involved in TRALI occurrence in predisposed patients. ${ }^{13}$ These findings suggest that older PLTs would increase the risk of developing TRALI. However, to our knowledge, no clinical studies have addressed this issue.

\section{Sepsis, organ failure and mortality}

Based on the "second hit model," it is hypothesized that critically ill patients who already have a coexisting inflammatory state could be more susceptible to adverse effects of stored PLTs. ${ }^{49}$ It is anticipated that an association between PLT storage duration and poor outcome would be easier to identify in this population than in stable patients who are also exposed to a lower volume of blood component administration. However, determining whether stored PLTs are as safe as fresh PLTs is difficult due to the numerous and different factors that influence potential study endpoints such as mortality, morbidity, and sepsis. Population, severity of underlying illness, other comorbidities, and other interventions all have an impact on these clinically centered outcomes. In addition, the large sample sizes required to reduce the feasibility of such studies.

Only two studies have investigated transfusion safety of PLTs stored up to 5 days, both in critically ill patient populations. ${ }^{50,51}$ Although none of them have reported an association between PLT storage duration and mortality, one study reported an association between administration of older PLTs and an increased risk of sepsis in 381 trauma patients (adjusted odds ratio for sepsis: $2.4,95 \%$ confidence interval 1.4-4.7, $P=0.02) .{ }^{51}$ Nonetheless, this was a single center, retrospective study with no clear criteria for defining sepsis, and the findings need to be confirmed in additional studies.

There is currently not enough evidence to support an association between adverse events and PLT storage duration when PLTs are stored for up to 5 days and studies that 
have investigated the efficacy of PLT stored for up to 7 days were underpowered to analyze any association between PLT storage duration and recipient outcomes, including morbidity and mortality. ${ }^{52,53,19,21}$

\section{Future research}

Assuming that extension of PLT storage duration would improve PLT availability and decrease PLT wastage, prospective research is required to confirm that PLTs stored for longer durations are as safe and effective as fresher PLTs, particularly in vulnerable patient populations.

\section{Conclusion}

Ensuring PLT availability while minimizing wastage is challenging, particularly at times of increased demand or in small and remote health services and non-civilian settings. Due to the increasing demand for PLTs observed internationally, and the high wastage rates, improving PLT inventory management should be a priority for blood services and health care systems. Alternatives to liquidstored PLTs are being evaluated, although currently there is insufficient data for routine use. Prolonged PLT storage duration (up to 5-7 days) is associated with accumulation of bioreactive substances and some studies suggest that these bioreactive substances could lead to inflammation activation in predisposed recipients. Nonetheless, clinical consequences of prolonged PLT storage duration have not been demonstrated. Changes in manufacturing processes of liquid-stored PLTs, bacterial contamination screening, and use of PRTs may mitigate some of the risks of stored PLTs. Larger prospective studies are warranted to confirm the benefit and safety of routinely implementing prolonged PLT storage duration.

\section{Disclosure}

The authors report no conflicts of interest in this work.

\section{References}

1. Holcomb JB, Tilley BC, Baraniuk S, et al. Transfusion of plasma, platelets, and red blood cells in a 1:1:1 vs a 1:1:2 ratio and mortality in patients with severe trauma: the PROPPR randomized clinical trial. JAMA. 2015;313(5):471-482.

2. Estcourt LJ, Stanworth SJ, Doree C, Hopewell S, Trivella M, Murphy MF. Comparison of different platelet count thresholds to guide administration of prophylactic platelet transfusion for preventing bleeding in people with haematological disorders after myelosuppressive chemotherapy or stem cell transplantation. Cochrane Database Syst Rev. 2015;11:CD010983.

3. Lieberman L, Bercovitz RS, Sholapur NS, Heddle NM, Stanworth SJ, Arnold DM. Platelet transfusions for critically ill patients with thrombocytopenia. Blood. 2014;123(8):1146-1151; quiz 280.
4. Bilgin YM, van de Watering LM, Versteegh MI, van Oers MH, Vamvakas EC, Brand A. Postoperative complications associated with transfusion of platelets and plasma in cardiac surgery. Transfusion. 2011;51(12):2603-2610.

5. Kaufman RM, Assmann SF, Triulzi DJ, Strauss RG, Ness P, Granger S, Slichter SJ. Transfusion-related adverse events in the Platelet Dose study. Transfusion. 2015;55(1):144-153.

6. Eder AF, Dy BA, Perez JM, Rambaud M, Benjamin RJ. The residual risk of transfusion-related acute lung injury at the American Red Cross (2008-2011): limitations of a predominantly male-donor plasma mitigation strategy. Transfusion. 2013;53(7):1442-1449.

7. Garraud O, Cognasse F, Tissot JD, et al. Improving platelet transfusion safety: biomedical and technical considerations. Blood Transfus. 2016;14(2):109-122.

8. Vlaar AP, Hofstra JJ, Kulik W, et al. Supernatant of stored platelets causes lung inflammation and coagulopathy in a novel in vivo transfusion model. Blood. 2010;116(8):1360-1368.

9. Aslam R, Speck ER, Kim M, Freedman J, Semple JW. Transfusionrelated immunomodulation by platelets is dependent on their expression of MHC Class I molecules and is independent of white cells. Transfusion. 2008;48(9):1778-1786.

10. Aubron C, Nichol A, Cooper DJ, Bellomo R. Age of red blood cells and transfusion in critically ill patients. Ann Intensive Care. 2013;3(1):2.

11. Estcourt LJ. Why has demand for platelet components increased? A review. Transfus Med. 2014;24(5):260-268.

12. Cognasse F, Hamzeh-Cognasse H, Lafarge S, et al. Donor platelets stored for at least 3 days can elicit activation marker expression by the recipient's blood mononuclear cells: an in vitro study. Transfusion. 2009;49(1):91-98.

13. Khan SY, Kelher MR, Heal JM, et al. Soluble CD40 ligand accumulates in stored blood components, primes neutrophils through CD40, and is a potential cofactor in the development of transfusion-related acute lung injury. Blood. 2006;108(7):2455-2462.

14. de Kort W, Janssen M, Kortbeek N, Jansen N, van der Wal J, van Dijk N. Platelet pool inventory management: theory meets practice. Transfusion. 2011;51(11):2295-2303.

15. van Dijk N, Haijema R, van der Wal J, Sibinga CS. Blood platelet production: a novel approach for practical optimization. Transfusion. 2009;49(3):411-420.

16. National Blood Authorities. BloodMove Platelets - a successful system to significantly reduce platelet wastage [BloodMove Platelets - a successful system to significantly reduce platelet wastage. Available from: https://www.blood.gov.au/bloodmove-platelets. Accessed June 15, 2016.

17. Glynn SA, Busch MP, Dodd RY, et al. Emerging infectious agents and the nation's blood supply: responding to potential threats in the 21 st century. Transfusion. 2013;53(2):438-454.

18. Dunbar NM, Dumont LJ, Szczepiorkowski ZM. How do we implement Day 6 and Day 7 platelets at a hospital-based transfusion service? Transfusion. 2016;58(6):1262-1266.

19. MacLennan S, Harding K, Llewelyn C, et al. A randomized noninferiority crossover trial of corrected count increments and bleeding in thrombocytopenic hematology patients receiving 2- to 5-versus 6- or 7-day-stored platelets. Transfusion. 2015;55(8):1856-1865; quiz 5.

20. Milford CE, Reade CM. Comprehensive review of platelet storage methods for use in the treatment of active hemorrhage. Transfusion. 2016;56(Suppl 2):S140-S148.

21. Dijkstra-Tiekstra MJ, Pietersz RN, Hendriks EC, Reesink HW, Huijgens PC. In vivo PLT increments after transfusions of WBC-reduced PLT concentrates stored for up to 7 days. Transfusion. 2004;44(3): 330-336.

22. Hay SN, Immel CC, McClannan LS, Brecher ME. The introduction of 7-day platelets: a university hospital experience. J Clin Apher. 2007;22(5):283-286.

23. Haijema R, van der Wal J, van Dijk NM. Blood platelet production: Optimization by dynamic programming and simulation. Comput Oper Res. 2007;34(3):760-779. 
24. Slichter SJ, Jones M, Ransom J, et al. Review of in vivo studies of dimethyl sulfoxide cryopreserved platelets. Transfus Med Rev. 2014;28(4):212-225.

25. Lelkens CC, Koning JG, de Kort B, Floot IB, Noorman F. Experiences with frozen blood products in the Netherlands military. Transfus Apher Sci. 2006;34(3):289-298.

26. Khuri SF, Healey N, MacGregor H, et al. Comparison of the effects of transfusions of cryopreserved and liquid-preserved platelets on hemostasis and blood loss after cardiopulmonary bypass. J Thorac Cardiovasc Surg. 1999;117(1):172-183; discussion 83-84.

27. Cap AP, Perkins JG. Lyophilized platelets: challenges and opportunities. J Trauma. 2011;70(Suppl 5):S59-S60.

28. Veihola M, Aroviita P, Linna M, Sintonen H, Kekomaki R. Variation of platelet production and discard rates in 17 blood centers representing 10 European countries from 2000 to 2002. Transfusion. 2006;46(6):991-995.

29. Gulliksson H. Platelet storage media. Vox Sang. 2014;107(3): 205-212.

30. Cardigan R, Sutherland J, Wadhwa M, Dilger P, Thorpe R. The influence of platelet additive solutions on cytokine levels and complement activation in platelet concentrates during storage. Vox Sang. 2003;84(1):28-35.

31. Lozano M CJ. Platelet concentrates: balancing between efficacy and safety. Presse Méd. 2016;45(7-8 Pt 2):e289-e298.

32. Kaplan A, Lindgren B, Marschner S, et al. Evaluation of the posttransfusion platelet increment and safety of riboflavin-based pathogen reduction technology (PRT) treated platelet products stored in platelet additive solution for 5 days or less versus 6-7 days. Transfus Apher Sci. 2016;54(2):248-252.

33. van der Meer PF, Pietersz RN, Reesink HW. Storage of platelets in additive solution for up to 12 days with maintenance of good in-vitro quality. Transfusion. 2004;44(8):1204-1211.

34. Tynngard N. Preparation, storage and quality control of platelet concentrates. Transfus Apher Sci. 2009;41(2):97-104.

35. Slichter SJ, Harker LA. Preparation and storage of platelet concentrates. II. Storage variables influencing platelet viability and function. $\mathrm{Br} J$ Haematol. 1976;34(3):403-419.

36. Thon JN, Schubert P, Devine DV. Platelet storage lesion: a new understanding from a proteomic perspective. Transfus Med Rev. 2008;22(4):268-279.

37. Ohto H, Nollet KE. Overview on platelet preservation: better controls over storage lesion. Transfus Apher Sci. 2011;44(3):321-325.

38. Wandall HH, Hoffmeister KM, Sorensen AL, Rumjantseva V, Clausen H, Hartwig JH, Slichter SJ. Galactosylation does not prevent the rapid clearance of long-term, 4 degrees C-stored platelets. Blood. 2008;111(6):3249-3256.
39. Picker SM, Speer R, Gathof BS. Functional characteristics of buffy-coat PLTs photochemically treated with amotosalen- $\mathrm{HCl}$ for pathogen inactivation. Transfusion. 2004;44(3):320-329.

40. Picker SM, Schneider V, Oustianskaia L, Gathof BS. Cell viability during platelet storage in correlation to cellular metabolism after different pathogen reduction technologies. Transfusion. 2009;49(11):2311-2318.

41. Dumont LJ, AuBuchon JP, Gulliksson $\mathrm{H}$, et al. In vitro $\mathrm{pH}$ effects on in vivo recovery and survival of platelets: an analysis by the BEST Collaborative. Transfusion. 2006;46(8):1300-1305.

42. Sarkodee-Adoo CB, Kendall JM, Sridhara R, Lee EJ, Schiffer CA. The relationship between the duration of platelet storage and the development of transfusion reactions. Transfusion. 1998;38(3):229-235.

43. Leach MF, AuBuchon JP. Effect of storage time on clinical efficacy of single-donor platelet units. Transfusion. 1993;33(8):661-664.

44. Lazarus HM, Herzig RH, Warm SE, Fishman DJ. Transfusion experience with platelet concentrates stored for 24 to 72 hours at 22 degrees C. Importance of storage time. Transfusion. 1982;22(1):39-43.

45. Hogge DE, Thompson BW, Schiffer CA. Platelet storage for 7 days in second-generation blood bags. Transfusion. 1986;26(2):131-135.

46. Agence Nationale de Sécurité du médicament ANSM et des Produits de Santé. Rapport d'activité d'Hémovigilance 2014. Available from: http://ansm.sante.fr/var/ansm_site/storage/original/application/4ee5a 6f35365ab8b2ab1ad5eaccb5bd6.pdf. Accessed June 15, 2016.

47. Hong H, Xiao W, Lazarus HM, Good CE, Maitta RW, Jacobs MR. Detection of septic transfusion reactions to platelet transfusions by active and passive surveillance. Blood. 2016;127(4):496-502.

48. Slichter SJ, Kaufman RM, Assmann SF, et al. Dose of prophylactic platelet transfusions and prevention of hemorrhage. $N$ Engl J Med. 2010;362(7):600-613.

49. Silliman CC. The two-event model of transfusion-related acute lung injury. Crit Care Med. 2006;34(Suppl 5):S124-S131.

50. Welsby IJ, Lockhart E, Phillips-Bute B, et al. Storage age of transfused platelets and outcomes after cardiac surgery. Transfusion. 2010;50(11):2311-2317.

51. Inaba K, Branco BC, Rhee P, et al. Impact of the duration of platelet storage in critically ill trauma patients. J Trauma. 2011;71(6):1766-1773; discussion 73-74.

52. Diedrich B, Ringden O, Watz E, Shanwell A. A randomized study of buffy coat platelets in platelet additive solution stored 1-5 versus 6-7 days prior to prophylactic transfusion of allogeneic haematopoietic progenitor cell transplant recipients. Vox Sang. 2009;97(3):254-259.

53. Dijkstra-Tiekstra MJ, van de Watering LM, Rondeel JM, Slomp J, de Wildt-Eggen J. Implementation of a new platelet pooling system for platelet concentrates led to a higher corrected count increment after transfusion: a comparative observational study of platelet concentrates before and after implementation. Transfus Med. 2014;24(2):99-104.
International Journal of Clinical Transfusion Medicine

\section{Publish your work in this journal}

International Journal of Clinical Transfusion Medicine is an international, peer-reviewed, open access, online journal publishing clinicalexperimental, policy-making and evidence-based practices of all topics pertaining to clinical transfusion medicine. Original research, short reports, reviews, case reports and commentaries are invited.

\section{Dovepress}

The manuscript management system is completely online and includes a very quick and fair peer-review system, which is all easy to use. Visit http://www.dovepress.com/testimonials.php to read real quotes from published authors. 